\title{
Optimal Demodulation of PAM Signals
}

\author{
JOHN B. MOORE AND PRITI HETRAKUL
}

\begin{abstract}
Kalman filtering theory is applied to yield an optimal causal demodulator for pulse-amplitude-modulated (PAM) signals in the presence of white Gaussian noise. The discrete-time data (or sampled continuous-time data) are assumed to be either a stationary or nonstationary Gaussian stochastic process, in general nonwhite.

Optimal demodulation with delay is also achieved by application of Kalman filtering theory. The resulting demodulators (fixed-lag smoothers) are readily constructed and their performance represents in many cases a significant improvement over that for the optimal demodulator without delay. The fixed-lag smoothing results are in contrast to those for amplitude-modulated signals (AM) where only approximate fixed-lag smoothing is possible, and this with considerable design effort.
\end{abstract}

The performance of the optimal PAM demodulator is shown to be equivalent to that of an optimal discrete filter for the discrete data.

\section{INTRODUCTION}

B ROADLY speaking, the problem to be considered in this paper is the optimal demodulation of pulseamplitude-modulated (PAM) signals in the presence of white Gaussian channel noise. The term "optimal" is used in the minimum-least-squares-error sense.

The conventional frequency-domain approaches to this problem [1], [2] yield the spectrum of the receiver (demodulator) for the unrealizable case when an infinite delay in demodulation is assumed. The frequency spectrum of the optimal receiver is not given explicitly, and complicated algorithms are presented for its calculation. Once this is obtained, standard techniques can then be employed to yield a realizable (causal) demodulator where a fixed time lag (the lag may be zero) exists between the signal production and its estimation. Of course the greater this lag, the more nearly optimal will be the system.

The problem considered in this paper can now be less broadly stated as the optimal fixed-lag demodulation of PAM signals.

The approach to this problem to be adopted throughout the paper is the state-variable approach. In particular, both continuous-time and discrete-time Kalman-filtering results are applied to a state-space PAM signal model to yield the various results. For those familiar with state-space methods and Kalman-filtering results and their application to communications system designs [3]-[5], the application of these methods is perfectly straightforward, although perhaps not immediately obvious.

One important advantage of using the state-variable approach in filtering problems is that time-varying signal models and receivers present no special difficulties. These time-varying systems cannot be handled without the in-

Manuscript received September 15, 1971; revised April 24, 1972. This work was supported by the Australian Research Grants Committee.

The authors are with the Department of Electrical Engineering, University of Newcastle, New South Wales, Australia. troduction of some simplifying assumptions using frequencydomain approaches since these are restricted to time-invariant systems. As for the PAM filtering problem under consideration, the signal model used in our paper is a timevarying one and we believe corresponds better to the practical situation than does the model of [3], [4]. As a consequence of the time-varying model, the optimal receiver is time varying, but we hasten to point out that the optimal time-invariant receiver can be determined if required.

A direct comparison of the results of this paper with the frequency-domain results is difficult, as the problems solved are posed somewhat differently. Since the solutions are optimum or near optimum in either case, comparison is perhaps best attempted by considering the calculations involved, the resulting optimum system structures, and the claims of optimality made for each approach.

Before proceeding with the development of the ideas of the paper, we present a precise problem statement. Consider the case of discrete data $y\left(t_{k}\right), k=1,2, \cdots$, which is a sample function of a discrete-time Gaussian stochastic process, which can be modeled as follows:

$$
\begin{aligned}
& x\left(t_{k}\right)=\phi\left(t_{k}, t_{k-1}\right) x\left(t_{k-1}\right)+u\left(t_{k-1}\right) \\
& y\left(t_{k}\right)=h^{\prime}\left(t_{k}\right) x\left(t_{k}\right)
\end{aligned}
$$

where $x\left(t_{k}\right)$ is a state $n$-vector and $u\left(t_{k}\right)$ is a white Guassian noise discrete process of zero mean and covariance $Q\left(t_{k}\right) \delta\left(t_{k}-t_{l}\right)$. The initial state vector is a zero-mean Gaussian random vector independent of $u(\cdot)$ and having a covariance $P_{0}$. It is now assumed that unknown noisy measurements $z(t)$ are made of a carrier signal $c(t)$ modulated by the data $y\left(t_{k}\right)$ as follows

$z(t)=c(t) y\left(t_{k}\right)+v(t), \quad t_{k} \leq t<t_{k+1}, k=1,2, \cdots$.

Here $c(t)$ is assumed to be periodic with period $t_{k+1}-$ $t_{k}=T$, while $v(t)$ is a zero-mean white Gaussian noise process independent of $u(\cdot)$ and $x\left(t_{0}\right)$, which has a covariance $r(t) \delta(t-\tau)$. The case of vector measurements is not discussed since to treat this case is but a minor extension.

We seek an optimal estimate of the data conditioned on the measurements as follows

$$
\hat{y}\left(t_{k} / t\right)=E\left[y\left(t_{k}\right) / z(\tau), \quad t_{k} \leq \tau<t\right] .
$$

For the case of PAM demodulation without delay, we require $t_{k} \leq t<t_{k+1}$. For the case of PAM demodulation with delay, we require $t_{l} \leq t<t_{l+1}$ where $l>k$.

Of course the discrete data $y\left(t_{k}\right)$ could be in fact samples taken from a continuous-time process. For the case when the continuous-time process can be modeled using a linear dynamical finite-dimensional system driven by white Gaussian noise, it is not difficult to determine the parameters of (1) and (2) from those of the continuous model [6]. 
In the next section both continuous-time and discretetime linear optimal filtering results are reviewed in order to introduce notation and for reference purposes. In the following section these results are applied to yield optimal PAM demodulators without delay. In Section IV, the case of optimal demodulation with delay is considered, while in Section $V$ further extensions are briefly discussed.

\section{Review of Linear Optimal Filtering Results}

\section{A. Continuous-Time Case}

Consider the linear dynamical system described by the differential equation

$$
\begin{aligned}
\dot{x}(t) & =F(t) x(t)+G(t) u(t) \\
z(t) & =H^{\prime}(t) x(t)+v(t)
\end{aligned}
$$

where $x(t)$ is the state $n$-vector, $z(t)$ the measurement $m$-vector, and inputs $u(t)$ and $v(t)$ are independent white Gaussian noise vectors of zero mean and covariances $Q(t) \delta(t-\tau)$ and $R(t) \delta(t-\tau)$, respectively. The matrices $F, G$, and $H$ are of appropriate dimension. The initial state vector $x\left(t_{0}\right)=x_{0}$ is a zero-mean Gaussian random vector independent of $u(\cdot)$ and $v(\cdot)$ and having a covariance $P_{0}$.

The optimal (minimum error variance) filter for the continuous system (5), (6) consists of the following differential equations for the conditional mean $\hat{x}(t / t)$ and error covariance matrix $P(t)$

$$
\dot{\hat{x}}(t / t)=F(t) \hat{x}(t / t)+K(t) \tilde{z}(t)
$$

with $\hat{x}(0 / 0)$ given

$$
\begin{aligned}
\tilde{z}(t)= & z(t)-H^{\prime}(t) \hat{x}(t / t) \\
K(t)= & P(t) H(t) R^{-1}(t) \\
\dot{P}(t)= & F(t) P(t)+P(t) F^{\prime}(t)-P(t) H(t) R^{-1}(t) H^{\prime}(t) P(t) \\
& +G(t) Q(t) G^{\prime}(t) \\
P\left(t_{0}\right)= & P_{0} .
\end{aligned}
$$

Of course the definitions of the conditional mean $\hat{x}(t / t)$ and error covariance $P(t)$ are $\hat{x}(t / t)=E[x(t) / z(\tau), 0 \leq$ $\tau<t]$ and $P(t)=E\left\{[x(t)-\hat{x}(t / t)][x(t)-\hat{x}(t / t)]^{\prime}\right\}$. In order to give relevant stability results we first define the following quantities

$$
\begin{gathered}
W\left(t_{1}, t_{0}\right)=\phi_{c}\left(t_{1}, t_{0}\right) P_{0} \phi_{c}{ }^{\prime}\left(t_{1}, t_{0}\right) \\
\quad+\int_{t_{0}}^{t_{1}} \phi_{c}\left(t_{1}, t\right) G(t) Q(t) G^{\prime}(t) \Phi_{c}{ }^{\prime}\left(t_{1}, t\right) d t \\
\dot{\phi}_{c}\left(t, t_{0}\right)=F(t) \phi_{c}\left(t, t_{0}\right) ; \quad \phi_{c}\left(t_{0}, t_{0}\right)=I .
\end{gathered}
$$

Now as established in [7], [11] with $F, H, Q$ and $R^{-1}$ bounded, with $\left[F, H R^{-1 / 2}\right]$ uniformly completely observable and with $W\left(t_{1}, t_{0}\right)$ nonsingular for some $t_{1}$ the optimal filter is asymptotically stable (but not necessarily exponentially asymptotically stable although this is guaranteed if $\left[F, G Q^{1 / 2}\right]$ is also uniformly completely controllable).

An example of the direct application of these results is to the optimal demodulation of amplitude-modulated stationary Gaussian signals in white Gaussian noise [3]-[5].
For this case $F$ and $G$ are constant and $H$ is time varying. The simplest case is double-sideband suppressed-carrier modulation (DSB-SC), where $H^{\prime}(t)=H^{\prime} \sin \omega_{c} t$ with $\omega_{c}$ the carrier frequency. Clearly the filtering results (7)-(9) can be specialized to this case. Actually, simplifying approximations can be made to the results for the usual case when the modulating signal is bandlimited and the carrier frequency is much greater than this message bandwidth. This is simply because the lowpass nature of the optimal filter ensures that the parameter variations at carrier frequency are filtered out. We see that the equations

$$
\begin{gathered}
\dot{\hat{x}}(t / t)=F \hat{x}(t / t)+P(t) H R^{-1} \tilde{z}(t) \sin \omega_{c} t \\
\tilde{z}(t)=z(t)-H^{\prime} \hat{x}(t / t) \sin \omega_{c} t \\
\dot{P}(t)=F P(t)+P(t) F^{\prime}-P(t) H R^{-1} H^{\prime} P(t) \sin ^{2} \omega_{c} t \\
+G Q G^{\prime}, \quad P(0)=P_{0}
\end{gathered}
$$

may be approximated by [3]

$$
\begin{aligned}
\hat{x}(t / t) & =F \hat{x}(t / t)+\bar{P} H R^{-1} \bar{z}(t) \\
\bar{z}(t) & =z(t) \sin \omega_{c} t-\frac{1}{2} H^{\prime} \hat{x}(t / t) \\
0 & =F \bar{P}+\bar{P} F^{\prime}-\frac{1}{2} \bar{P} H R^{-1} H^{\prime} \bar{P}+G Q G^{\prime} .
\end{aligned}
$$

These equations define a stationary filter.

A ready extension to the preceding results is possible when the carrier is gated with a binary signal as in some forms of PAM. These extensions are not considered here since this form of PAM, although often discussed in textbooks, is not usually implemented in practice.

\section{B. Discrete-Time Case}

Consider the vector difference equation

$$
\begin{aligned}
& x\left(t_{k}\right)=\phi_{d}\left(t_{k}, t_{k-1}\right) x\left(t_{k-1}\right)+u\left(t_{k-1}\right) \\
& z\left(t_{k}\right)=H^{\prime}\left(t_{k}\right) x\left(t_{k}\right)+v\left(t_{k}\right)
\end{aligned}
$$

where $k=1,2, \cdots$ and $x\left(t_{k}\right)$ is the $n$-vector state, $\phi_{d}\left(t_{k}, t_{k-1}\right)$ the nonsingular state transition matrix, and the vectors $u\left(t_{k}\right)$ and $v\left(t_{k}\right)$ are independent white Gaussian noise sequences of zero mean and having covariances $Q\left(t_{k}\right) \delta\left(t_{k}-t_{l}\right)$ and $R\left(t_{k}\right) \delta\left(t_{k}-t_{l}\right)$, respectively. The initial state vector $x\left(t_{0}\right)=x_{0}$ is a zero-mean Gaussian random vector, independent of $u(\cdot)$ and $v(\cdot)$ and having a covariance $P_{0}$.

The optimal (minimum error variance) filter for the discrete system (11), (12), consists of the following difference equations for the conditional mean $\hat{x}\left(t_{k} / t_{k}\right)=E\left[x\left(t_{k}\right) / z\left(t_{l}\right)\right.$, $1 \leq l \leq k]$ and error covariance matrix $P\left(t_{k}\right)=E\left\{\left[x\left(t_{k}\right)-\right.\right.$ $\left.\left.\hat{x}\left(t_{k} / t_{k}\right)\right]\left[x\left(t_{k}\right)-\hat{x}\left(t_{k} / t_{k}\right)\right]^{\prime}\right\}$

$$
\begin{aligned}
\hat{x}\left(t_{k} / t_{k-1}\right)= & \phi_{d}\left(t_{k}, t_{k-1}\right) \hat{x}\left(t_{k-1} / t_{k-1}\right) \\
\hat{x}\left(t_{k} / t_{k}\right)= & \hat{x}\left(t_{k} / t_{k-1}\right)+K\left(t_{k}\right) \tilde{z}\left(t_{k}\right) \\
\tilde{z}\left(t_{k}\right)= & z\left(t_{k}\right)-H^{\prime}\left(t_{k}\right) \hat{x}\left(t_{k} / t_{k-1}\right) \\
K\left(t_{k}\right)= & P\left(t_{k} / t_{k-1}\right) H\left(t_{k}\right)\left[H^{\prime}\left(t_{k}\right) P\left(t_{k} / t_{k-1}\right) H\left(t_{k}\right)\right. \\
& \left.+R\left(t_{k}\right)\right]^{-1}
\end{aligned}
$$

$P\left(t_{k} / t_{k-1}\right)=\phi_{d}\left(t_{k}, t_{k-1}\right) P\left(t_{k-1} / t_{k-1}\right) \phi_{d}^{\prime}\left(t_{k}, t_{k-1}\right)+Q\left(t_{k-1}\right)$ 


$$
\begin{aligned}
P\left(t_{k} / t_{k}\right) & =\left[P^{-1}\left(t_{k} / t_{k-1}\right)+H\left(t_{k}\right) R^{-1}\left(t_{k}\right) H^{\prime}\left(t_{k}\right)\right]^{-1} \\
& =\left[I-K\left(t_{k}\right) H^{\prime}\left(t_{k}\right)\right] P\left(t_{k} / t_{k-1}\right) .
\end{aligned}
$$

Manipulation of these equations yield alternative expressions for the filter as

$$
\begin{aligned}
\hat{x}\left(t_{k} / t_{k}\right)= & {\left[I-K\left(t_{k}\right) H^{\prime}\left(t_{k}\right)\right] \phi_{d}\left(t_{k}, t_{k-1}\right) \hat{x}\left(t_{k-1} / t_{k-1}\right) } \\
& +K\left(t_{k}\right) z\left(t_{k}\right) \\
= & P\left(t_{k} / t_{k}\right) P^{-1}\left(t_{k} / t_{k-1}\right) \phi_{d}\left(t_{k}, t_{k-1}\right) \hat{x}\left(t_{k-1} / t_{k-1}\right) \\
& +K\left(t_{k}\right) z\left(t_{k}\right) .
\end{aligned}
$$

Uniform complete controllability and uniform complete observability of the discrete system (11) and (12) together with the boundedness of $\phi, H, Q$, and $R^{-1}$ ensures uniform exponential asymptotic stability of the filter (19) for the limiting case as $k$ approaches infinity [7], [11].

\section{Optimal PaM Demodulation-Without Delay}

The first step in our derivation of an optimal PAM demodulator without delay is a key step. It is to construct a signal process model of (PAM) from (1)-(3) amenable to the application of the filtering theory reviewed in the previous section.

We consider the signal process model of Fig. 1(a), which is described by the following equations.

$$
\begin{aligned}
\dot{x}(t) & =0 \\
z(t) & =c(t) h^{\prime}\left(t_{k}\right) x(t)+v(t)
\end{aligned}
$$

for $t_{k} \leq t<t_{k+1}$ and $k=1,2, \cdots$. To complete the model we include the relationship

$$
x\left(t_{k}\right)=\phi\left(t_{k}, t_{k-1}\right) x\left(t_{k-1}\right)+u\left(t_{k-1}\right) .
$$

Now the solution of (20) is $x(t)=x\left(t_{k}\right)$, for $t_{k} \leq t<t_{k+1}$ and $k=1,2, \cdots$, and this substitution of (20) into (21) and application of (2) yields

$$
z(t)=c(t) y\left(t_{k}\right)+v(t)
$$

and thus equations (20), (21), and (1) represent a valid PAM signal process model.

Application of the continuous-time filtering theory reviewed in the previous section to this model of Fig. 1(b) now yields the following equations for the conditional mean $\hat{x}(t / t)$ and covariance $P(t)$

$$
\begin{aligned}
\dot{\hat{x}}(t / t) & =k(t) \tilde{z}(t) \\
\tilde{z}(t) & =z(t)-c(t) h^{\prime}\left(t_{k}\right) \hat{x}(t / t) \\
k(t) & =P(t) h\left(t_{k}\right) c(t) r^{-1}(t) \\
\dot{P}(t) & =-c^{2}(t) r^{-1}(t) P(t) h\left(t_{k}\right) h^{\prime}\left(t_{k}\right) P(t),
\end{aligned}
$$

where $t_{k} \leq t<t_{k+1}$, and $k=1,2, \cdots$. These equations follow directly from (7)-(10) by direct substitution of the parameters $G(t)=0, F(t)=0, H(t)=c(t) h\left(t_{k}\right)$, which are extracted from the signal process representation equations (20) and (21). Notice that $\hat{x}\left(t_{k} / t_{k}\right)$ and $P\left(t_{k}\right)$, for $k=$ $1,2, \cdots$ are not defined from these equations. We show in the next two paragraphs that they may, however, be

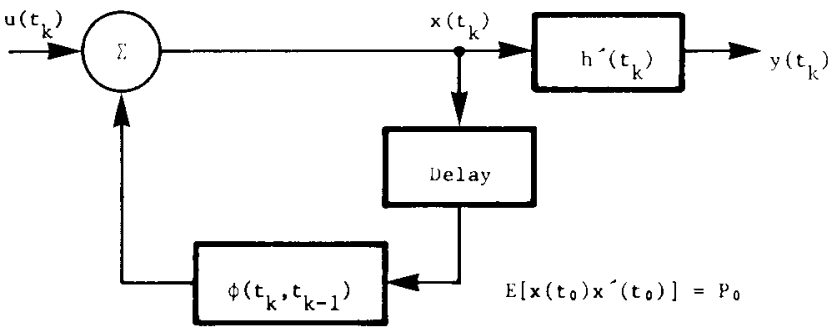

(a)

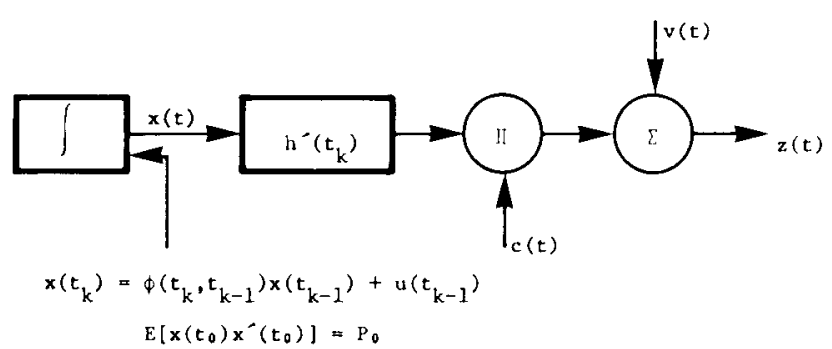

(b)

Fig. 1. (a) Discrete message model. (b) Representation of noisy observations of PAM signal for $t_{k} \leq t<t_{k+1}$ and $k=1,2, \cdots$. (Note equations for resetting the state $x(\cdot)$ at instants $t_{k}$ ).

determined using their definitions together with (I) for $k=1,2, \cdots$ as

$$
\begin{aligned}
\hat{x}\left(t_{k} / t_{k}\right) & =\phi\left(t_{k}, t_{k-1}\right) \hat{x}\left(t_{k}{ }^{-} / t_{k}{ }^{-}\right) \\
P\left(t_{k}\right) & =\phi\left(t_{k}, t_{k-1}\right) P\left(t_{k}^{-}\right) \phi^{\prime}\left(t_{k}, t_{k-1}\right)+Q\left(t_{k-1}\right)
\end{aligned}
$$

where $\hat{x}\left(t_{\overline{1}} / t_{\bar{T}}\right)=0$ and $P\left(t_{\bar{T}}\right)=P_{0}$. The notation $X\left(t_{\bar{k}}\right)$ is used to denote $X\left(t_{k}-\varepsilon\right)$ for arbitrarily small $\varepsilon>0$.

The proof of (26) is as follows. Taking the expectations of both sides of (1) conditioned on the measurements $z(\tau)$, $t_{1} \leq \tau<t_{k}$ yields

$$
\begin{aligned}
\hat{x}\left(t_{k} / t_{k}\right) & =\phi\left(t_{k}, t_{k-1}\right) \hat{x}\left(t_{k-1} / t_{k}\right) \\
& =\phi\left(t_{k}, t_{k-1}\right) \hat{x}\left(t_{k}{ }^{-} / t_{k}\right) \\
& =\phi\left(t_{k}, t_{k-1}\right) \hat{x}\left(t_{k}{ }^{-} / t_{k}{ }^{-}\right) .
\end{aligned}
$$

The first equality holds since $E\left[u\left(t_{k}\right) / z(\tau), t_{1} \leq \tau<t_{k+1}\right]=$ 0 . To see this observe that the measurements $z(\tau), t_{1} \leq$ $\tau<t$ depend only on the noise $v(\tau), t_{1} \leq \tau<t$ and the states $x\left(t_{l}\right), 1 \leq l \leq k$, both of which are independent of $u\left(t_{k}\right)$. The second equality holds since $x\left(t_{k-1}\right)=x\left(t_{k}{ }^{-}\right)$ [from (20)] and the final equality holds by virtue of the definition of the conditional expectation.

The proof of (27) follows from the definition of $P\left(t_{k}\right)$ and the application of (1) and (26) as follows.

$$
P\left(t_{k}\right)=E\left\{\left[x\left(t_{k}\right)-\hat{x}\left(t_{k} / t_{k}\right)\right]\left[x\left(t_{k}\right)-\hat{x}\left(t_{k} / t_{k}\right)\right]^{\prime}\right\} .
$$

The error term $\left[x\left(t_{k}\right)-\hat{x}\left(t_{k} / t_{k}\right)\right]$ may be rewritten using (1) and (26) as

$x\left(t_{k}\right)-\hat{x}\left(t_{k} / t_{k}\right)=\phi\left(t_{k}, t_{k-1}\right)\left[x\left(t_{k-1}\right)-\hat{x}\left(t_{k}{ }^{-} / t_{k}{ }^{-}\right)\right]+u\left(t_{k-1}\right)$.

This is the sum of two independent Gaussian random variables and therefore the covariance of the sum is the sum of the covariances, namely (27).

The optimal demodulator for PAM signals is therefore 
given by (22)-(27). For two alternative forms for the realization of the demodulator, see Fig. 2. The error covariance is given explicitly by (25) and (27). We comment that this is like a continuous-time Kalman filter except that the integrators are reset at the instants $t_{k}$.

We have so far seen that the structure of the optimal demodulator is in the form of a Kalman filter with its integrators reset at the time instants $t_{k}$. We now go on to show that the performance and stability properties of this filter at the time instants $t_{k}$ are identical to those for a discrete Kalman filter driven from noisy measurements of the unmodulated discrete message signal. In particular, we show that the covariance of the noise for this discrete case is a discrete covariance $\bar{r}\left(t_{k}\right) \delta\left(t_{k}-t_{l}\right)$ defined from

$$
\bar{r}^{-1}(t)=\int_{t_{k}}^{t} c^{2}(\tau) r^{-1}(\tau) d \tau, \quad t_{k} \leq t<t_{k+1} .
$$

As a first step to deriving this result we consider the transition matrix $\phi_{C L}$ associated with the closed-loop PAM demodulator (22), (23). Its equation is

$$
\dot{\phi}_{C L}\left(t, t_{k}\right)=-P(t) h\left(t_{k}\right) c(t) r^{-1}(t) c(t) h^{\prime}\left(t_{k}\right) \phi_{C L}\left(t, t_{k}\right)
$$

for $t_{k} \leq t<t_{k+1}$, where of course $\phi_{C L}\left(t_{k}, t_{k}\right)=I$. Using this equation together with (25), it is not hard to show that

$$
{ }_{d t}^{d}\left[P^{-1}(t) \phi_{C L}\left(t, t_{k}\right)\right]=0
$$

and thus that

$$
\phi_{C L}\left(t, t_{k}\right)=P(t) P^{-1}\left(t_{k}\right)
$$

where $t_{k} \leq t<t_{k+1}$. Now by virtue of (26) and the definition of $\phi_{C L}\left(t, t_{k}\right)$ we have

$$
\begin{aligned}
& \phi_{C L}\left(t_{k+\overline{1}}, t_{\bar{k}}\right)=\phi_{C L}\left(t_{k+1}^{-}, t_{k}\right) \phi\left(t_{k}, t_{k-1}\right) \\
& \phi_{C L}\left(t_{k+\overline{1}}, t_{\bar{k}}\right)=P\left(t_{k+1}^{-}\right) P^{-1}\left(t_{k}\right) \phi\left(t_{k}, t_{k-1}\right) .
\end{aligned}
$$

At this stage we simply comment that this equation is very like that for the transition matrix $P\left(t_{k} / t_{k}\right) P^{-1}\left(t_{k} / t_{k-1}\right)$. $\phi_{d}\left(t_{k}, t_{k-1}\right)$ of the discrete filter of (19).

As a second step to relating the PAM demodulator (22), (23), to a discrete filter we consider the explicit solution of the Riccati equation (25). From (25)

$$
\begin{aligned}
& \dot{P}^{-1}(t)=c^{2}(t) r^{-1}(t) h\left(t_{k}\right) h^{\prime}\left(t_{k}\right) \\
& P^{-1}(t)=\int_{t_{k}}^{t} c^{2}(\tau) r^{-1}(\tau) h\left(t_{k}\right) h^{\prime}\left(t_{k}\right) d \tau+P^{-1}\left(t_{k}\right)
\end{aligned}
$$

and thus

$$
\begin{aligned}
P(t) & =\left[P^{-1}\left(t_{k}\right)+h\left(t_{k}\right) \bar{r}^{-1}(t) h^{\prime}\left(t_{k}\right)\right]^{-1} \\
& =P\left(t_{k}\right)\left[I-h\left(t_{k}\right) \bar{k}^{\prime}(t)\right]
\end{aligned}
$$

where we have used the Schur matrix identity, the definitions (27)-(28), and the definition

$$
\bar{k}(t)=P\left(t_{k}\right) h\left(t_{k}\right)\left[r(t)+h^{\prime}\left(t_{k}\right) P\left(t_{k}\right) h\left(t_{k}\right)\right]^{-1}
$$

for $t_{k} \leq t<t_{k+1}$. These results lead to

$$
\begin{aligned}
P\left(t_{k+1}^{-}\right) & =\left[P^{-1}\left(t_{k}\right)+h\left(t_{k}\right) \bar{r}^{-1}\left(t_{k+1}^{-}\right) h^{\prime}\left(t_{k}\right)\right]^{-1} \\
& =\left[I-\bar{k}\left(t_{k+1}^{-}\right) h^{\prime}\left(t_{k}\right)\right] P\left(t_{k}\right) .
\end{aligned}
$$

Clearly (27), (31), and (32) may be solved recursively to obtain $P\left(t_{k}\right)$ and $P\left(t_{k}{ }^{-}\right)$for $k=0,1, \cdots$ without the need to explicitly calculate $P(t)$, for $t_{k} \leq t<t_{k+1}$. Furthermore, these recurrence relationships have a one-to-one correspondence with (17), (16), and (18) for the discrete filtering problem. The one-to-one correspondence between (29) and (19) is also now in context. The various correspondences are as follows.

$$
\begin{aligned}
P\left(t_{k+1}^{-}\right) & \sim P\left(t_{k} / t_{k}\right) \\
\phi\left(t_{k}, t_{k-1}\right) & \sim \phi_{d}\left(t_{k} / t_{k-1}\right) \\
h\left(t_{k}\right) & \sim H\left(t_{k}\right) \\
\hat{x}\left(t_{k+1}^{-} / t_{k+1}^{-}\right) & \sim \hat{x}\left(t_{k} / t_{k}\right) \\
P\left(t_{k}\right) & \sim P\left(t_{k} / t_{k-1}\right) \\
\bar{r}\left(t_{k+1}^{-}\right) & \sim R\left(t_{k}\right) \\
\bar{k}\left(t_{k+1}^{-}\right) & \sim K\left(t_{k}\right) .
\end{aligned}
$$

From a study of the one-to-one correspondence just mentioned, we conclude that the performance and stability. properties of an optimal PAM demodulator are identical to those for optimal filtering of the noisy measurements of the unmodulated discrete signal where the noise is discrete with covariance $\bar{r}\left(t_{k}\right) \delta\left(t_{k}-t_{l}\right)$. That is, the optimal PAM demodulator is guaranteed to be exponentially asymptotically stable for the case when $\phi, h, Q$, and $r^{-1}$ are bounded and the system (1) and (2) is uniformly completely controllable and uniformly completely observable.

The case of a time-invariant, completely observable, and completely controllable message model with a stationary noise process is of interest. For this case $\phi\left(t_{k}, t_{k-1}\right)=\phi$, $h\left(t_{k}\right)=h, Q(t)=Q$, and $r(t)=r$, where $\phi, h$, and $r$ are constant. As for the discrete Kalman-filter recurrence equations, for the limiting case as $k$ becomes infinite, the recurrence equations (27) and (25) have solutions independent of $k$. Denoting these solutions as

$$
\bar{P}=\lim _{k \rightarrow \infty} P\left(t_{k}\right) \quad \bar{P}=\lim _{k \rightarrow \infty} P\left(t_{k}{ }^{-}\right)
$$

it is clear that these may be determined from the following algebraic equations

$$
\begin{aligned}
& \bar{P}=\left[\bar{P}^{-1}+h \bar{r}^{-1} h^{\prime}\right]^{-1}=\bar{P}\left[I-h \bar{k}^{\prime}\right] \\
& \bar{P}=\phi \bar{P} \phi^{\prime}+Q
\end{aligned}
$$

where, with $T$ the time interval between measurement data,

$$
\bar{r}^{-1}(t)=r^{-1} \int_{0}^{t} c^{2}(\tau) d \tau \quad \bar{k}(t)=\bar{P} h\left[\vec{r}(t)+h^{\prime} \bar{P} h\right]^{-1}
$$

for $0 \leq t<T, \bar{r}=\bar{r}\left(T^{-}\right)$, and $\bar{k}=\bar{k}\left(T^{-}\right)$.

The solution $P(t)$ is calculated using a specialization of 


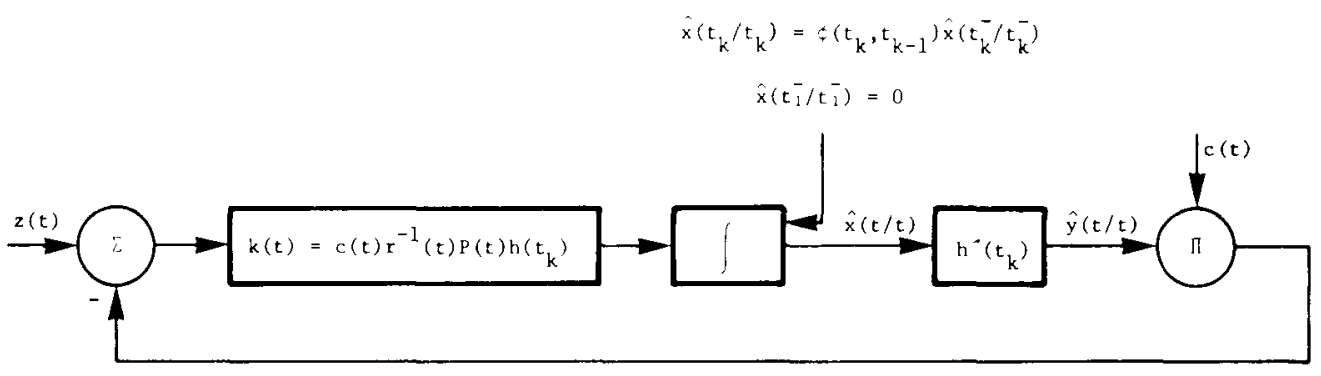

(a)

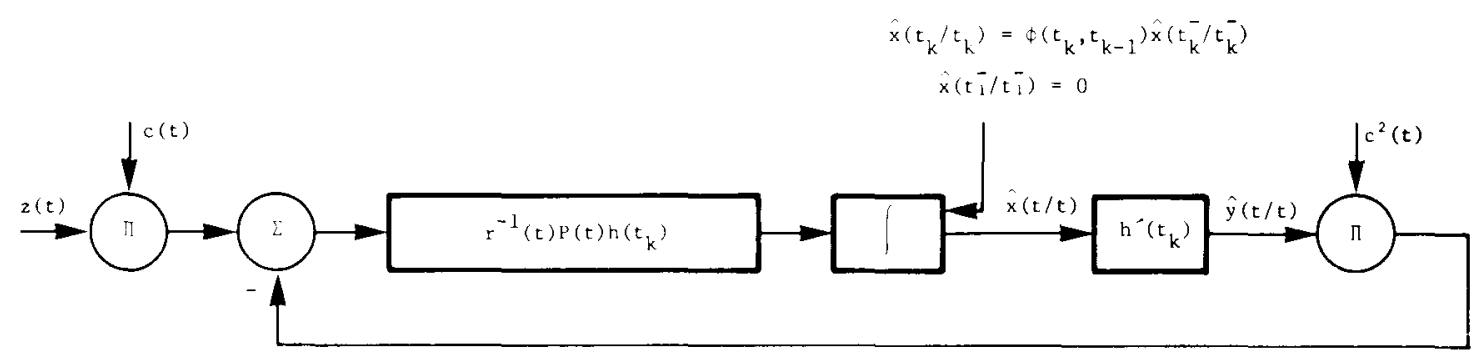

(b)

Fig. 2. Alternative forms of the optimal PAM demodulator for $t_{k} \leq t<t_{k+1}$ and $k=1,2, \cdots$.

(28) as

$$
P(t)=\left[\bar{P}^{-1}+h \bar{r}^{-1}(t) h^{\prime}\right]^{-1}=\bar{P}\left[I-h \bar{k}^{\prime}(t)\right]
$$

for $0 \leq t<T$. Clearly, the calculations involved and the construction of an optimal PAM demodulator are considerably more straightforward for the case when the message is a stationary random process.

In order to illustrate the usage of $(31)-(33), \bar{P}$ is calculated as a function of $\bar{r}^{-1}$ for the scalar message model case and the results plotted in Fig. 3. These results can be compared with those obtained using frequency-domain techniques in [1].

So far, our demodulation results are for an arbitrary periodic carrier signal $c(t)$ where $c(t)$ is periodic with period $t_{k+1}-t_{k}$. Once the carrier $c(t)$ is specified simplifying approximations can usually be made. For example, for the case of double-sideband suppressed-carrier (DSB-SC), PAM modulation, $c(t)=c \sin \omega_{c} t$ and $c^{2}(t)=\left(c^{2} / 2\right)-$ $\left(c^{2} / 2\right) \cos 2 \omega_{c} t$. Now, since as indicated in Fig. 2(b), $c^{2}(t)$ is injected into a loop with low-pass filter characteristics inside the loop, the effects of the $\left(c^{2} / 2\right) \cos 2 \omega_{c} t$ term for the usual case when $2 \omega$, is large can be ignored. This allows the simplification as indicated in Fig. 4, which is drawn for the stationary message and noise case.

A major drawback of the optimal PAM demodulator derived previously is the inclusion of the time-varying gain element whose value depends on the solution of multidimensional Riccati equation. In what follows we shall consider the optimal time-invariant PAM demodulator for the case of a time-invariant message model and stationary noise. The optimal demodulator will be assumed to have the structure indicated in Fig. 4, where the gain element $k$, is given by $k_{f}=P_{x} h \bar{r}^{-1}$ for some $n \times n$ matrix $P_{x}$.

The steady-state error covariance $\bar{P}_{s}$ associated with the time-invariant filter of Fig. 4 can be shown to be the solu- tion of

$$
e^{-A T} \bar{P}_{s} e^{-A^{\prime} T}=\phi \bar{P}_{s} \phi^{\prime}+Q+\int_{0}^{T} e^{-A \tau} k_{f} \bar{r} k_{f}^{\prime} e^{-A \tau} d \tau
$$

where $A=-(T / 2) k_{f} h^{\prime}$. The optimal time-invariant demodulator gain element $k_{f}$ is now determined by selecting $P_{\alpha}$ to minimize $\bar{P}_{s}$, or equivalently to minimize $\operatorname{tr}\left[\bar{P}_{s}\right]$. Results have been calculated for the case of a one-dimensional message model. These are indicated in Fig. 5 in terms of a scalar quantity $x$ defined from the following:

$$
P_{x}=\left[\begin{array}{c}
\bar{P}^{-1}+\alpha \bar{r}^{-1} T h^{2} \\
2
\end{array}\right]^{-1}
$$

Notice that when $\alpha=0, P_{x}=\bar{P}$, and when $\alpha=1, P_{x}=P$. The plots on Fig. 5 indicate that the optimal value of $\alpha$ is $x \simeq 0.5$ or equivalently that the optimal value of $P_{x}$ is $P_{x} \simeq\left[\bar{P}^{-1}+\left(\bar{r}^{-1} T h^{2}\right) / 4\right]^{-1}$.

\section{Optimal Demodulation of PaM Signals with Delay}

It is well known that the performance of a demodulator can be improved if the estimation of the message at any instant $t$ is based on signal measurements after $t$ as well as on measurements prior to time $t$ rather than on measurements prior to time $t$ alone. Fixed-lag smoothing of data, or equivalently demodulation with a fixed delay, is in fact an "on-line" estimation process in which estimation of the message at time $t$ is based on noisy measurements of the modulated signal up to time $(t+\Delta)$, where $\Delta$ is a positive time interval. There is thus a fixed delay $\Delta$ between the sending of a modulated signal and its demodulation.

The improvement in performance achicved using demodulation with an infinite delay (the unrealizable case) is not difficult to derive and is given, at least for linear problems of optimal AM demodulation, in various texts. The 


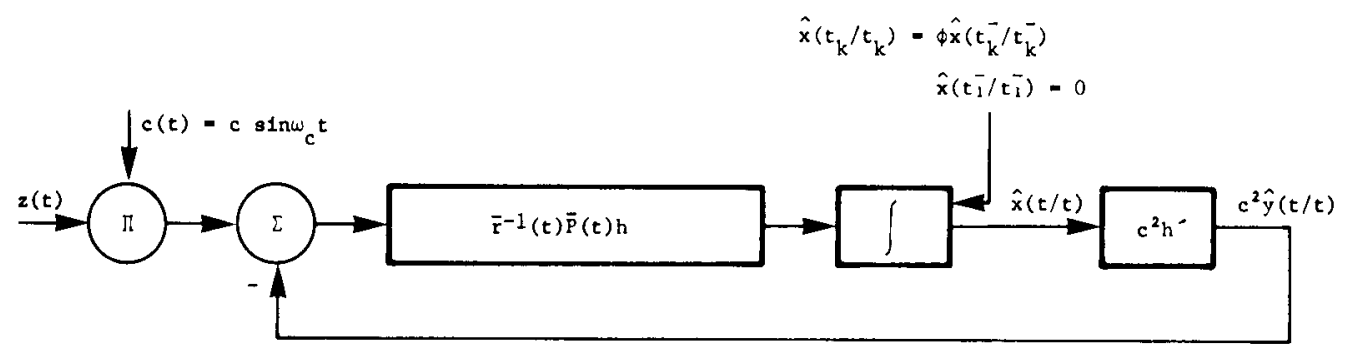

Fig. 3. Simplified optimal PAM demodulation for the stationary message and noise case when $(t)=\left(s \sin ()_{t} t\right.$. (The DSB-SC case.) For optimal time-varying filter $k_{f}(t)=r^{-1}(t) h \bar{P}(t)$. For the optimal time-invariant filter $k_{f}=r^{-i} h_{P_{x}}$.

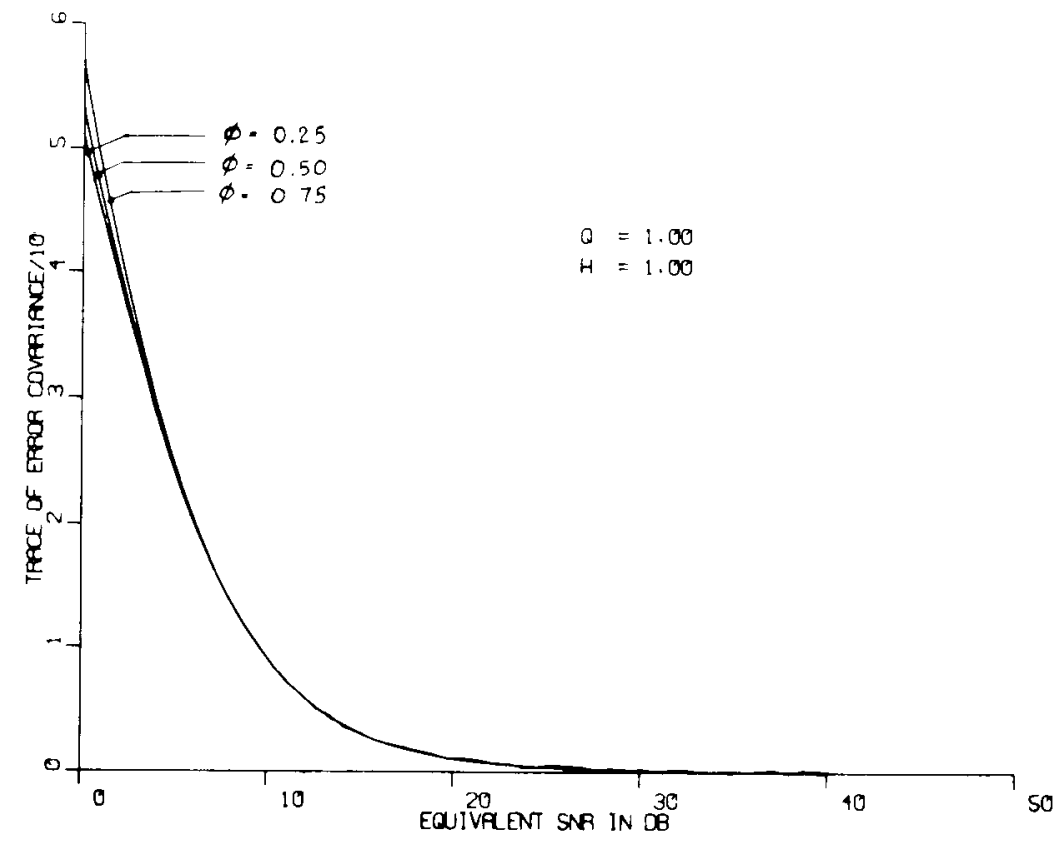

Fig. 4. Error versus SNR in decibels.

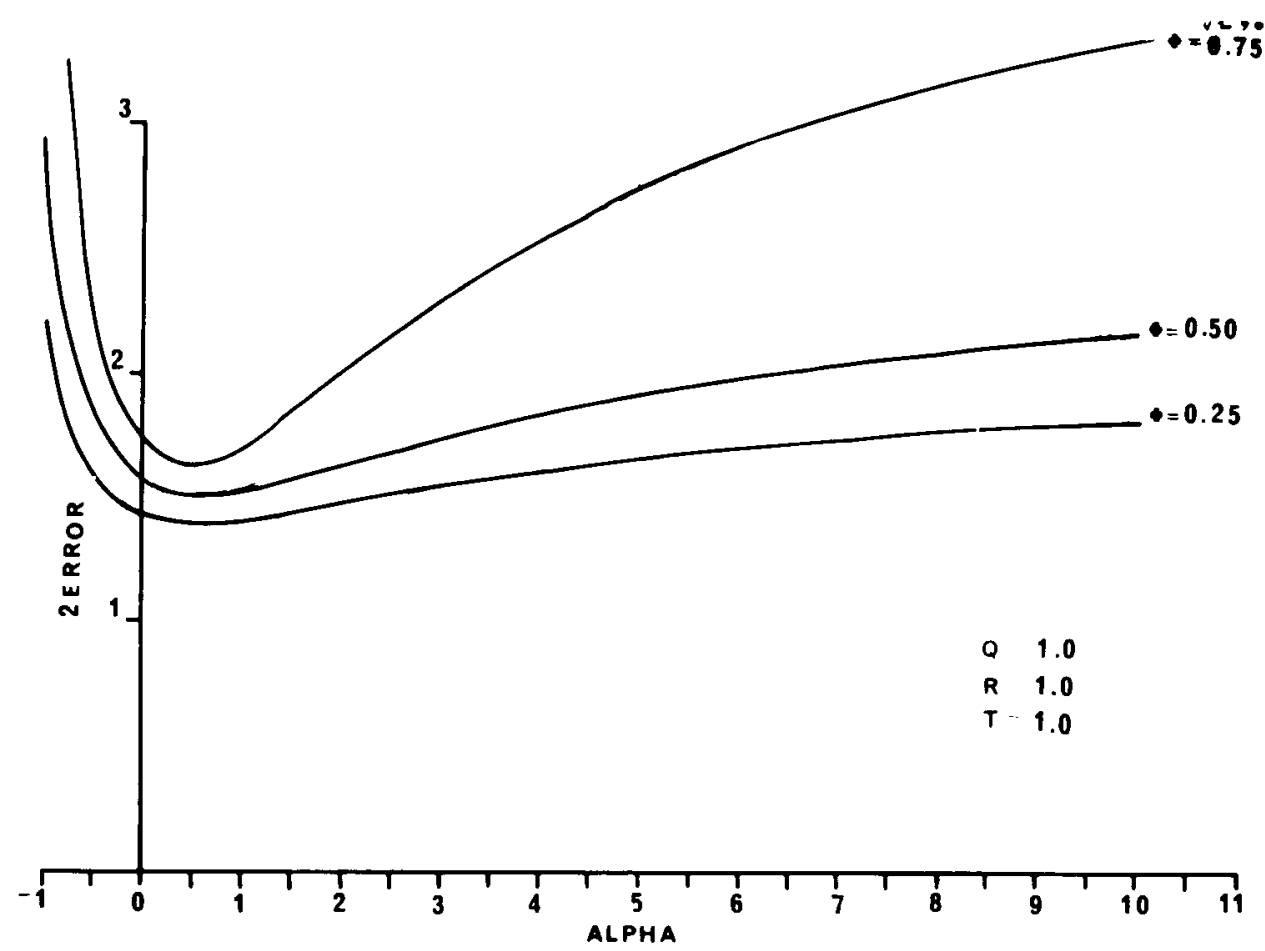

Fig. 5. Time-invariant filter performance. 
general problem of demodulation with a finite delay is, in filtering-theory terms, a nonlinear fixed-lag smoothing problem, which is one step more difficult than the nonlinear filtering problem for which only approximate solutions exist in other than the simplest of cases. Even the case of linear fixed-lag smoothing is difficult for continuous systems and only approximate finite-dimensional (suboptimal) smoothers can be achieved [8], [12]. ${ }^{1}$ This problem is currently being investigated.

The case of PAM demodulation is the one important demodulation problem for which optimal (as opposed to suboptimal) fixed-lag demodulators can be constructed. As we indicate in this section, these optimal PAM demodulators with finite delay can be derived by applications of Kalman linear filtering theory to an augmented signal process model. The key idea behind the derivations of this section is that the fixed-lag smoothed estimate of a signal is in fact nothing other than the filtered estimate of the signal delayed by the amount of the fixed lag but still conditioned on the noisy measurements of the undelayed signal. In considering demodulation of PAM with delay it is convenient to choose a delay equal to an integer number (say $N$ ) of time intervals of the discrete message process.

As a first step then in achieving a fixed-lag smoothed estimate, we consider the construction of a signal process model which includes the signal delayed by the fixed lag. For the PAM case, such a signal process model is the original model of the previous section augmented with integrators as indicated in Fig. 6 and the following equations

$$
\begin{aligned}
& {\left[\begin{array}{c}
\dot{x}_{0}(t) \\
\dot{x}_{1}(t) \\
\vdots \\
\dot{x}_{N}(t)
\end{array}\right]=0_{N n}} \\
& {\left[\begin{array}{c}
x_{0}\left(t_{k}\right) \\
x_{1}\left(t_{k}\right) \\
\vdots \\
x_{N}\left(t_{k}\right)
\end{array}\right]=\left[\begin{array}{rrrr}
\phi\left(t_{k+1}, t_{k}\right) & 0 & \cdots & 0 \\
& & & 0 \\
& I & & \\
& & & 0
\end{array}\right]} \\
& {\left[\begin{array}{c}
x_{0}\left(t_{k-1}\right) \\
x_{1}\left(t_{k-1}\right) \\
\vdots \\
x_{N}\left(t_{k-1}\right)
\end{array}\right]+\left[\begin{array}{c}
I \\
0 \\
\vdots \\
0
\end{array}\right] u\left(t_{k-1}\right)} \\
& z(t)=c(t)\left[h^{\prime}\left(t_{k}\right) 0 \cdots 0\right]\left[\begin{array}{c}
x_{0}\left(t_{k}\right) \\
x_{1}\left(t_{k}\right) \\
\vdots \\
x_{N}\left(t_{k}\right)
\end{array}\right]+\left[\begin{array}{c}
I \\
0 \\
\vdots \\
0
\end{array}\right] v(t),
\end{aligned}
$$

where $t_{k} \leq t<t_{k+1}, k=1,2, \cdots$, and $E\left[x\left(t_{0}\right) x^{\prime}\left(t_{0}\right)\right]=P_{0}$. Observe that the solution of $(35)$ is

$$
x_{i}(t)=x_{i}\left(t_{k}\right)=x_{0}\left(t_{k-i}\right)
$$

for $i=0,1, \cdots, N, t_{k} \leq t<t_{k+1}$, and $(k-i)=1,2, \cdots$. The important consequence of this equation for us here is that taking the expectation of (38) conditioned on the

\footnotetext{
${ }^{1}$ Recent studies indicate that optimal stable fixed-lag smoothers can be realized using switching networks and delay elements.
}
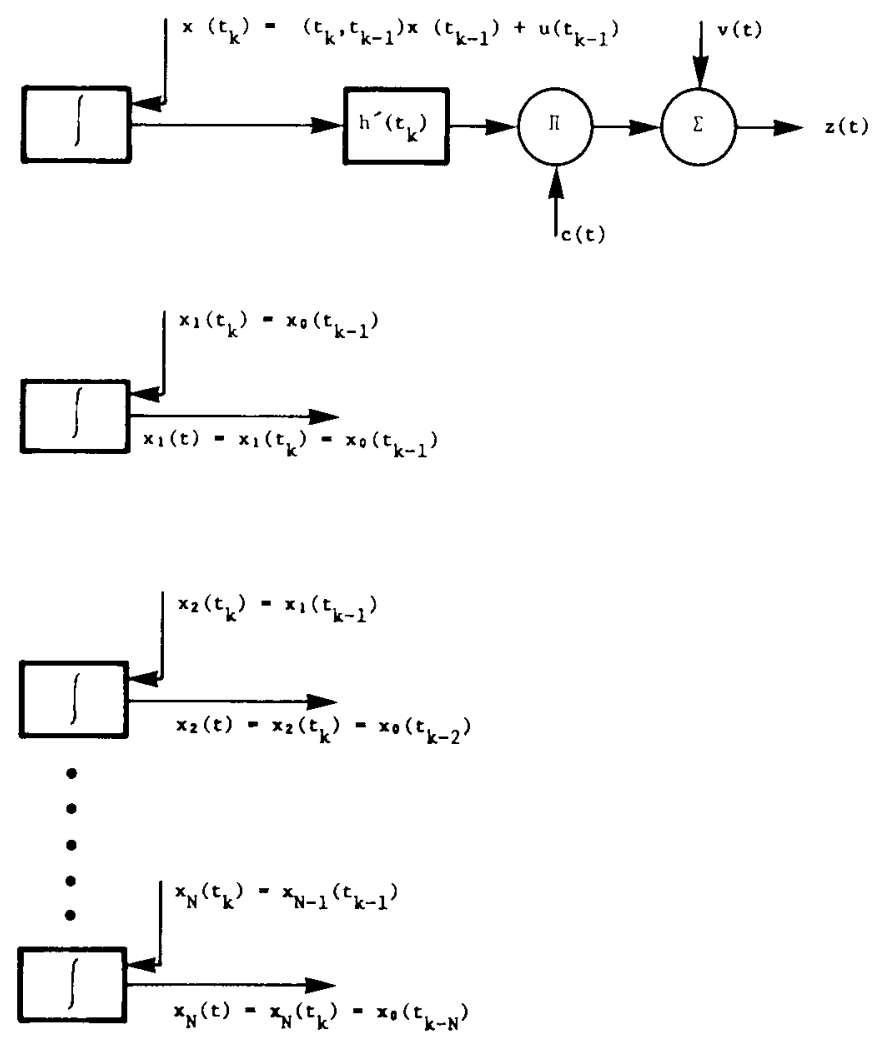
Fig. 6. Augmented signal process model. Note that $t_{k} \leq t<t_{k+1}$,
\[ k=0,1, \cdots \]

measurements up until time $t$ yields

$$
\hat{x}_{i}(t / t)=\hat{x}_{i}\left(t_{k} / t\right)=\hat{x}_{0}\left(t_{k-i} / t\right) \text {. }
$$

In other words, the desired fixed-lag smoothed estimate $\hat{x}_{0}\left(t_{k-N} / t\right)$ is equivalent to the filtered estimate $\hat{x}_{N}(t / t)$. To obtain $\hat{x}_{N}(t / t)$ we simply apply the filtering theory of the previous section to the augmented signal process model (35)-(37)

Defining the covariance of the error signal $\left\{\left[x_{0}{ }^{\prime}(t)-\right.\right.$ $\left.\left.\hat{x}_{0}{ }^{\prime}(t / t)\right]\left[x_{1}{ }^{\prime}(t)-\hat{x}_{1}{ }^{\prime}(t / t)\right] \cdots\left[x_{N}{ }^{\prime}(t)-\hat{x}_{N}{ }^{\prime}(t / t)\right]\right\}^{\prime}$ to be

$$
\left[\begin{array}{cccc}
P_{00} & P_{10}{ }^{\prime} & \cdots & P_{N 0}^{\prime} \\
P_{10} & P_{11} & & \\
\vdots & & \ddots & \vdots \\
P_{N 0} & \cdots & & P_{N N}
\end{array}\right]
$$

we now give the equations of the optimal filter for the augmented signal process model as

$$
\begin{aligned}
\dot{\hat{x}}_{i}(t / t) & =k_{i}(t) \tilde{z}(t) \\
\tilde{z}(t) & =z(t)-c(t) h^{\prime}\left(t_{k}\right) \hat{x}_{0}(t / t) \\
k_{i}(t) & =P_{i 0}(t) h\left(t_{k}\right) c(t) r^{-1}(t) \\
\bar{k}_{i}(t) & =P_{i 0}(t) h\left(t_{k}\right)\left[\bar{r}(t)+h^{\prime}\left(t_{k}\right) P_{00}\left(t_{k}\right) h\left(t_{k}\right)\right]^{-1} \\
& =P_{i 0}(t) P_{00}^{-1}(t) \bar{k}_{0}(t) \\
P_{i 0}(t) & =P_{i 0}\left(t_{k}\right)\left[I-h\left(t_{k}\right) \bar{k}_{0}{ }^{\prime}(t)\right] \\
P_{i i}(t) & =P_{i i}\left(t_{k}\right)-P_{i 0}\left(t_{k}\right) h\left(t_{k}\right) \bar{k}_{i}^{\prime}(t)
\end{aligned}
$$

for $i=0,1, \cdots, N$ and 


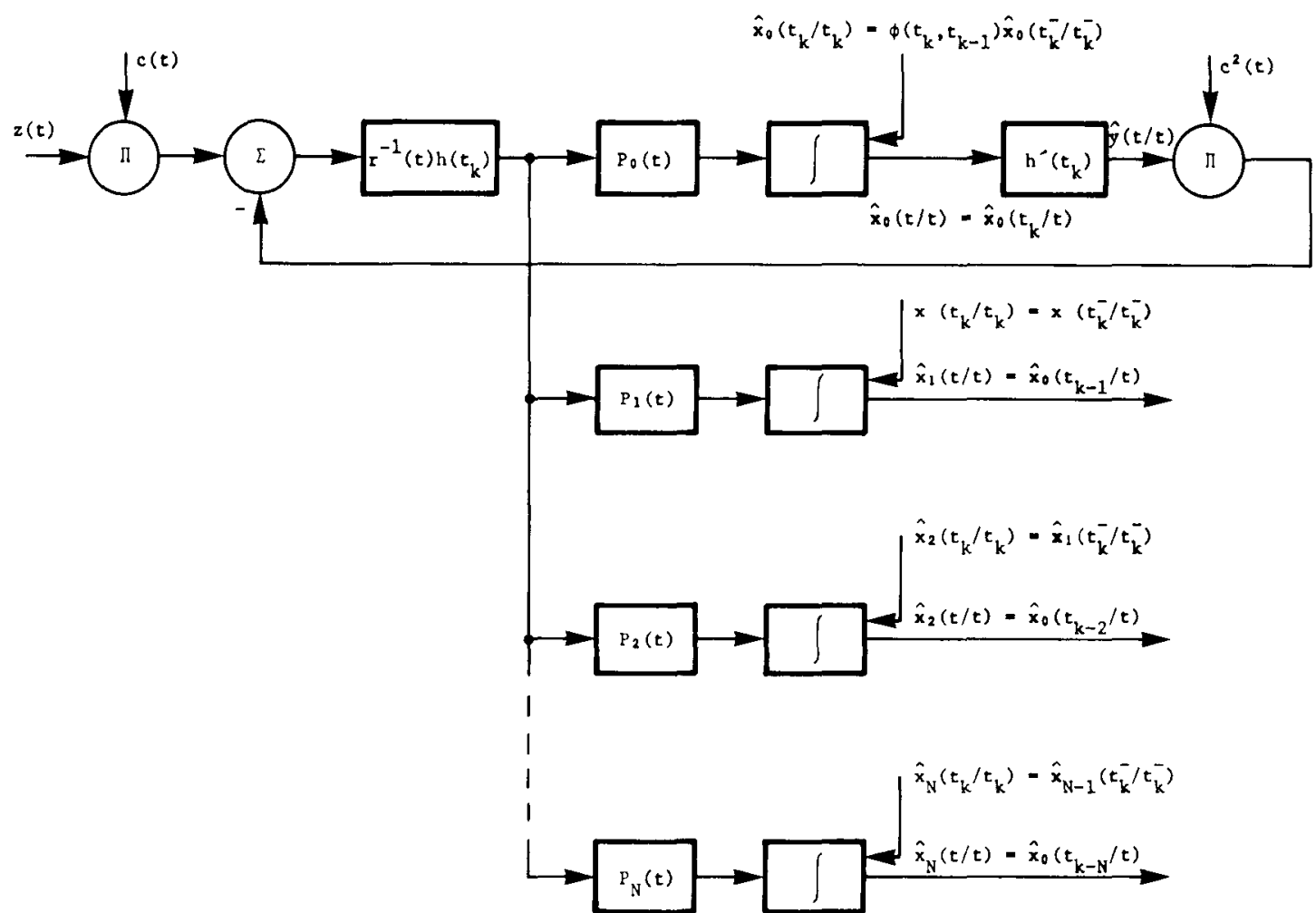

Fig. 7. Optimal PAM demodulator with delay for $t_{k} \leq t<t_{k+1}$ and $k=1,2, \cdots$.

$$
\begin{aligned}
\hat{x}_{0}\left(t_{k}, t_{k}\right) & =\phi\left(t_{k}, t_{k-1}\right) \hat{x}_{0}\left(t_{k}{ }^{-} / t_{k} \cdots\right) \\
\hat{x}_{i}\left(t_{k}, t_{k}\right) & =\hat{x}_{i-1}\left(t_{k}{ }^{-} / t_{k}{ }^{-}\right) \\
P_{00}\left(t_{k}\right) & =\phi\left(t_{k}, t_{k-1}\right) P\left(t_{\bar{k}}\right) \phi^{\prime}\left(t_{k}, t_{k-1}\right)+Q\left(t_{k-1}\right) \\
P_{i 0}\left(t_{k}\right) & =P_{i-1,0}\left(t_{k}{ }^{-}\right) \phi^{\prime}\left(t_{k}, t_{k-1}\right) \\
P_{i i}\left(t_{k}\right) & =P_{i-1, i-1}\left(t_{k}^{-}\right)
\end{aligned}
$$

for $i=1,2, \cdots, N$. These equations are all valid for $k=$ $1,2, \cdots$ and $t_{k} \leq t<t_{k+1}$ and together with (39) describe the optimal PAM demodulator with a delay of $N$ discrete time intervals, see also Fig. 7.

The error covariance $P_{N N}(t)$ in fact is the covariance of the error $\left[x_{N}(t)-\hat{x}_{N}(t / t)\right]=\left[x_{0}\left(t_{k-N}\right)-\hat{x}_{0}\left(t_{k-N} / t\right)\right]$ for $t_{k} \leq t<t_{k+1}$. A formula for $P_{N v}\left(t_{k+1}\right)$ indicating that $\left[P_{00}\left(t_{k-N}\right)-P_{N N}\left(t_{k}\right)\right]$, the error covariance reduction due to smoothing, is nonnegative, can be derived from the above equations as follows:

$$
\begin{aligned}
& P_{00}\left(t_{k \cdots N}\right)-P_{N N}\left(t_{k}\right)=\sum_{j=1}^{l} P_{N \cdot j, 0}\left(t_{k-j}\right) h\left(t_{k-j}\right)\left[\bar{r}\left(t_{\bar{k}+1 \cdots j}\right)\right. \\
& \left.+2 h^{\prime}\left(t_{k-j}\right) P_{00}\left(t_{h-j}\right) h\left(t_{k} j\right)\right]{ }^{1} \\
& \text { - } h^{\prime}\left(t_{k} j\right) P_{i-j, 0}^{\prime}\left(t_{k} \quad j\right) \\
& \geq 0 \text {. }
\end{aligned}
$$

These results correspond to smoothing improvement formulas for a purely discrete signal process model as in the ane of demodulation without delay. Discrete fixud lag stomethine resule of $|9|$ are also relevant.

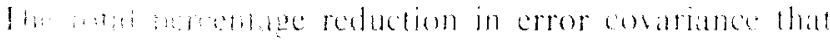

is possible from fixed-lag smoothing for the first-order model case is about 50 percent. This is achieved in the highsignal-to-noise-ratio situation. An even greater improvement is possible for higher order signal models with a sharp falloff in frequency characteristics. Each problem requires its own calculations.

A further comment on improvement due to smoothing is relevant. If the fixed lag is chosen to be $2 \tau$, say, where $\tau$ is the time constant of the optimal filter, then effectively as much improvement as is possible from smoothing is achieved.

To conclude this section, we stress the fact that optimal demodulators with a fixed delay for PAM signals can be achieved using an optimal demodulator without delay together with a few additional elements.

\section{CONCILIDING RIMARKS}

The results of the previous sections can be extended to less straightforward situations or specialized to known results. Some of these will now be discussed.

\section{A. Channel With Memory}

Such may arise when the chamnel is for example a transmission line. For this case we consider the signal process model as indicated in Fig. 8 . Its equations are as follows

$$
\begin{aligned}
\left|\begin{array}{c}
\dot{x}(t) \\
\dot{r}_{1}(t)
\end{array}\right| & =\left|\begin{array}{cc}
0 & 0 \\
(t) g_{1} h^{\prime}\left(t_{k}\right) & r_{1}
\end{array}\right|\left|\begin{array}{c}
x(t) \\
\gamma_{1}(t)
\end{array}\right| \\
(t) & =\left|\begin{array}{ll}
0 & h_{1}^{\prime}
\end{array}\right| \begin{array}{c}
x^{\prime}(t) \\
r_{1}(t)
\end{array} \mid .
\end{aligned}
$$




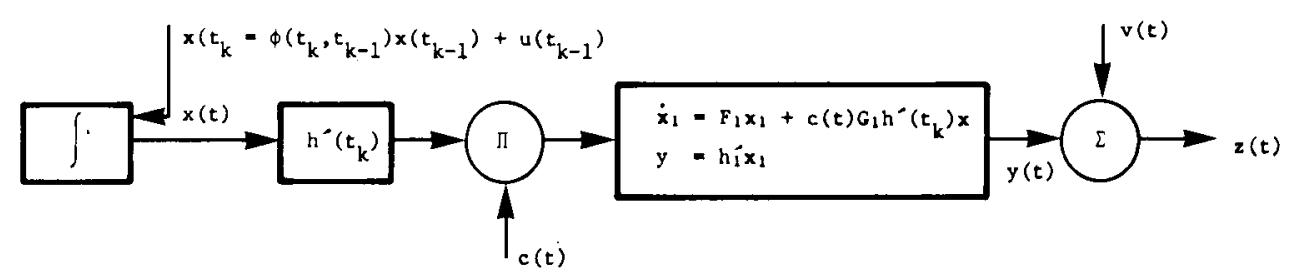

Fig. 8. Signal process model with channel memory for $t_{k} \leq t<t_{k+1}$ and $k=1,2, \cdots$.

The theory for this case is not difficult to develop along the lines of that in the previous sections. The multipath problem is a little more difficult and is a study in its own right. Intersymbol interference results can be extracted from studies of these more involved problems.

\section{B. The Colored-Noise Case}

If the channel noise included colored noise as well as white noise, a whitening filter could be constructed at the input to the receiver. Using this construction, the channel for the colored noise case is viewed as a channel with memory and white noise disturbances as above.

\section{The Case When $\phi\left(t_{k}, t_{k-1}\right)=0$}

When the message is uncorrelated from instant to instant (that is, the message is white noise) then of course $\phi\left(t_{k+1}, t_{k}\right)=0$ and the equations simplify to the case frequently treated in textbooks [10]. Each received pulse is demodulated without reference to the previous data. The results of this paper are clearly an improvement on these known results since in many cases of practical importance the assumption $\phi\left(t_{k+1}, t_{k}\right)=0$ is a severe one.

\section{Companded PAM}

Frequently, in a practical PAM system a nonlinearity is included in the modulator to limit the pulse amplitude. Optimal demodulation in this instance requires the application of nonlinear filtering theory and will be discussed in a companion paper dealing with optimal demodulation of pulse-modulated signals when the modulation is nonlinear, such as in pulse-frequency modulation (PFM).

\section{E. Incoherent Demodulation}

The case when the carrier signal is sinusoidal but of unknown phase at the receiver cannot be treated using linear filtering theory, since usually the phase of the carrier at the receiver is assumed to be a random variable uniformly distributed in the range $[0,2 \pi)$. The details will not be considered here. We comment, however, that for the low-noise case, the receiver of Fig. 3 can be modified to involve envelope detection with essentially no loss in performance. The modifications correspond to those indicated in the texts (see, for example, [10]) for the simpler receivers in which $\phi\left(t_{k}, t_{k-1}\right)=0$.

\section{F. Preemphasis Filtering}

Frequently, improved system performance can be achieved by inserting a filter between the message signal and the modulator. As far as the theory of this paper is concerned, this preemphasis filter is simply an augmentation of the message model and can be incorporated without undue difficulty. It appears that there is no explicit formula for the optimum preemphasis filter using the theory of the paper, and so it is suggested that standard search procedures be used to optimize the preemphasis filter parameters. The search is of course subject to bandwidth and power constraints.

\section{REFERENCES}

[1] T. Berger and D. W. Tufts, "Optimum pulse amplitude modulation, Part I: transmitter-receiver design and bounds from information theory," IEEE Trans. Inform. Theory, vol. IT-13, pp. 196-208, Apr. 1967.

[2] D. Chan and R. W. Donaldson, "Optimum pre- and postfiltering of sampled signals with application to pulse modulation and data compression systems," IEEE Trans. Commun. Technol., vol. COM-19, pp. 141-157, Apr. 1971.

[3] D. L. Snyder, The State-Variable Approach to Continuous Estimation. Cambridge, Mass.: M.I.T. Press, 1969

[4] H. L. Van Trees, Detection, Estimation and Modulation Theory, Part 1. New York: Wiley, 1968; also Part II. New York: Wiley, 1970.

[5] A. B. Baggeroer, State Variable and Communication Theory. Cambridge, Mass.: M.I.T. Press, 1970.

[6] A. H. Jazwinski, Stochastic Processes and Filtering Theory. New York: Academic Press, 1970, pp. 198-200.

[7] R. E. Kalman and R. S. Bucy, "New results in linear filtering and prediction theory," J. Basic' Eng., Trans. ASME, pp. 95-108, Mar. 1961

[8] S. Chirarattananon and B. D. O. Anderson, "The fixed-lag smoother as a stable finite-dimensionallinear system," Automatica, vol. 7, Nov. 1971 .

[9] J. B. Moore, "Discrete-time fixed-lag smoothing algorithms," Automatica, to be published.

[10] J. J. Stiffler, Theory of Synchronous Communications, Englewood Cliffs, N.J.: Prentice-Hall, 1970.

[11] B. D, O. Anderson, "Stability properties of Kalman-Bucy filters," J. Franklin Inst., vol. 291, pp. 137-144, Feb. 1971.

[12] C. N. Kelly and B. D. O. Anderson, "On the stability of fixed-lag smoothing algorithms," J. Franklin Inst., vol. 291, pp. 271-281, Apr. 1971. 